\title{
Systematic review of the predictive effect of MSI status in colorectal cancer patients undergoing 5FU-based chemotherapy
}

\author{
Elizabeth M Webber, Tia L Kauffman, Elizabeth O'Connor and Katrina AB Goddard ${ }^{*}$
}

\begin{abstract}
Background: We systematically reviewed the evidence for the interaction of microsatellite instability status (MSI) and treatment with 5FU in colorectal cancer to determine how well MSI status predicts health outcomes in patients undergoing 5FU-based chemotherapy.

Methods: We conducted a search of four electronic databases through June 2013. We considered studies that included both colorectal cancer patients treated with 5FU-based chemotherapy and untreated patients with survival outcomes presented by MSI status.

Results: We identified 16 studies for qualitative analysis (9,212 patients) with 14 studies eligible for meta-analysis. The microsatellite stable (MSS) group showed an effect of 5FU treatment on disease-free survival (HR of 0.62 [95\% Cl: 0.54, 0.71]) and overall survival (HR of 0.65 [95\% Cl: 0.54, 0.79]), indicating that MSS patients who received 5FU treatment had longer survival than MSS patients who were untreated. The effect of $5 \mathrm{FU}$ treatment was not statistically significant for microsatellite high (MSI-H) patients for disease-free survival (HR of 0.84 [95\% Cl: $0.53,1.32]$ ) or overall survival (HR 0.66 [95\% Cl: 0.43, 1.03]). However, the summarized point estimates of the effects of $5 \mathrm{FU}$ treatment for the MSS and MSI-H groups were not different at a statistically significant level.

Conclusions: Our analyses indicate that treatment with 5FU-based chemotherapy improves disease-free and overall survival in CRC patients, but that there is no difference in the effect of treatment based on MSI status. Therefore, the use of MSI status to guide treatment decisions about the use of $5 F U$ treatment for CRC has no significant benefits for patients.
\end{abstract}

Keywords: Genetic, Pharmacogenomic, Meta analysis, Lynch syndrome

\section{Background}

Colorectal cancer (CRC) is the third most commonly diagnosed cancer in the United States; about 1 in 20 Americans will develop CRC in their lifetime. Although the death rate from CRC has been dropping, it remains the third leading cause of cancer-related deaths [1]. About 15\% of CRC tumors develop via a pathway characterized by defective function of the DNA mismatch repair (MMR) system. MMR deficiency most commonly occurs through epigenetic inactivation of the MLH1 gene in sporadic CRCs, but can also occur through inherited mutations in any one of four genes (i.e.,

\footnotetext{
* Correspondence: Katrina.ab.goddard@kpchr.org

Center for Health Research - Kaiser Permanente Northwest, $3800 \mathrm{~N}$ Interstate Avenue, Portland, OR 97227, USA
}

(c) 2015 Webber et al.; licensee BioMed Central. This is an Open Access article distributed under the terms of the Creative Commons Attribution License (http://creativecommons.org/licenses/by/4.0), which permits unrestricted use, distribution, and reproduction in any medium, provided the original work is properly credited. The Creative Commons Public Domain Dedication waiver (http://creativecommons.org/publicdomain/zero/1.0/) applies to the data made available in this article unless otherwise stated.

MLH1, MSH2, MSH6, and PMS2). Tumors with MMR deficiency exhibit a high frequency of microsatellite instability (MSI-H) because these regions of the genome are particularly unstable and susceptible to errors that do not get corrected because of the defective MMR system [2]. For determining MSI status, the National Cancer Institute (NCI) recommends a microsatellite panel (NCI panel) consisting of two mononucleotide repeats (BAT26 and A4725) and three dinucleotide repeats (D5S346, D2S123, and D17S250). Using the NCI panel, MSI-H tumors are defined as having instability in two or more markers, and tumors with low or stable microsatellite instability have instability in one or no markers [3]. Tumor MMR status is also determined by immunohistochemical (IHC) analysis of the protein products of genes 
involved in DNA MMR, such as MLH1, MSH2, and MSH6, and PMS2 [4].

In advanced stage CRC patients, the fluoropyrimidine 5 -fluorouracil (5FU) has been the most widely used chemotherapeutic agent since the late 1950s [5]. 5FU, alone or in combination with other drugs, is recommended for first-line treatment in Stage III, Stage IV, and high risk Stage II CRC [6]. Recently, there has been some suggestion that MSI-H status, from either hereditary or acquired causes, could potentially guide treatment decisions for this very commonly used chemotherapeutic agent [7]. The expected effect of using MSI status to guide treatment decisions is to withhold treatment with 5FU for individuals who are unlikely to respond or to use alternative chemotherapies. Clinical practice guidelines provide conflicting recommendations about the use of MSI status to guide 5FU treatment decisions. Three major cancer guideline groups have stated that use of MSI for predicting response to therapy is not recommended [8-10]. Alternatively, updated guidelines for 2013 from the National Comprehensive Cancer Network recommend MSI testing for all patients with Stage II disease because MSI positive patients may have a good prognosis and do not benefit from $5 \mathrm{FU}$ therapy [6].

There is substantial data on the relationship between MSI status and prognosis in CRC patients. A metaanalysis with 7,642 cases demonstrated that patients with MSI-H tumors have a significantly better prognosis than those with MSS tumors [7]. This prognostic benefit of MSI-H status can confound the relationship between MSI and treatment effects, especially when only treated patients are considered. Figure 1 demonstrates that MSI-H patients appear to have better survival, even in the absence of a treatment benefit; their improved survival comes from the prognostic benefit of their MSI status rather than an effect of treatment.

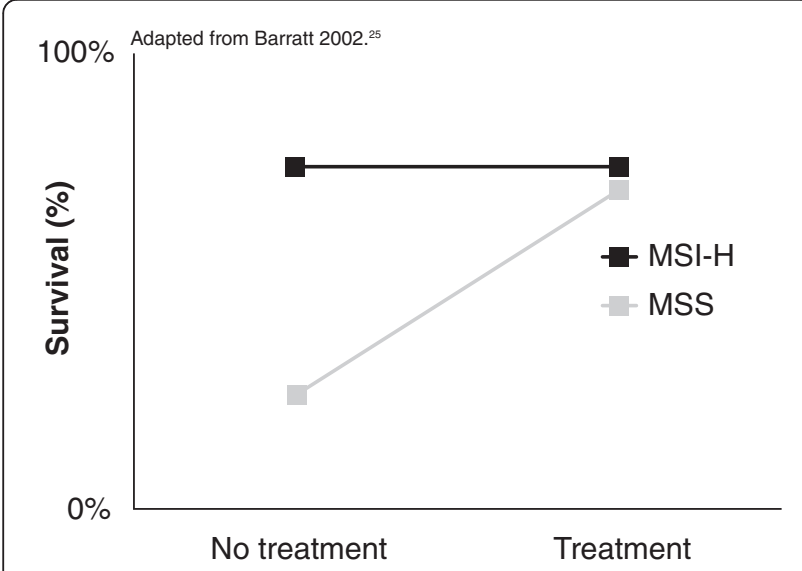

Figure $1 \mathrm{MSI}$ status is a prognostic and predictive marker. Adapted from Barratt 2002 [26].
We systematically reviewed the evidence for the interaction of microsatellite instability status (MSI) and treatment with 5FU in colorectal cancer to determine how well MSI status predicts health outcomes in patients undergoing 5FU-based chemotherapy.

\section{Methods \\ Eligibility criteria}

We considered studies that included both CRC patients treated with 5FU-based chemotherapy and untreated patients with survival outcomes presented by MSI status. We accepted either MSI or IHC testing to indicate MSI status. While any treatment containing 5FU therapy was included, we required that $>90 \%$ of patients received $5 \mathrm{FU}$ in the treatment group for inclusion of the study. Eligible study designs included randomized controlled or controlled clinical trials and prospective or retrospective cohort studies. We excluded studies that did not report disease free survival (DFS) or overall survival (OS) outcome separately for patients based on MSI and treatment status (or where data could not be calculated).

\section{Identification of studies}

Systematic literature searches were performed in the following databases through June 27, 2013: MEDLINE, PubMed, Cochrane Database of Systematic Reviews, and Cochrane Central Register of Controlled Trials. The search strategy was built to comprehensively capture the literature related to MSI and chemotherapeutic agents in the context of CRC treatment. Search details are provided in the supplementary materials (see Additional file 1: Search Strategy). The database searching was supplemented by the review of reference lists from existing reviews, hand searching selected scientific conferences from 2010-2013, and a search of ongoing and recently completed studies on clinicaltrials.gov.

Identified abstracts were independently reviewed by two investigators against inclusion criteria specified $a$ priori. Full-text articles of abstracts meeting inclusion criteria were dual-reviewed against the inclusion criteria. Disagreements were resolved through consensus or the input of a third reviewer.

We reviewed studies for the independence of their study populations. In the case where multiple studies were identified with population overlap, we included the study that presented the needed data with the largest number of participants.

\section{Quality assessment and data abstraction}

At least two investigators independently assessed the quality of each included study using questions adapted from the QUADAS-2 (Table 1) [11]. Overall quality codes were determined by discussion and consensus between the two reviewers. Quality categories of good, fair+, fair-, and poor 
Table 1 Article quality rating

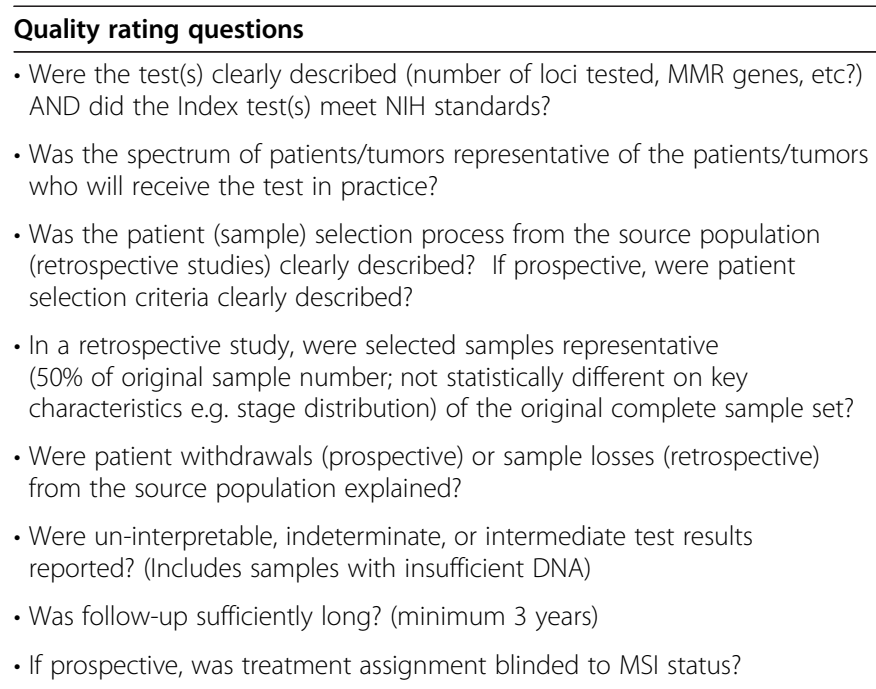

were used to indicate the relative risk of bias or concerns about applicability of each study. Despite the limitations of categorizing studies in this manner [12], these categories were used for ease of grouping. Studies that were considered poor quality were excluded from the analysis.

Data related to study design, population characteristics, chemotherapy use, MSI testing method and completeness, and relevant outcomes were abstracted from included studies into evidence tables with dual data checking by a second reviewer. Disagreements were resolved through consensus or input of a third reviewer.

Studies were included in the analysis regardless of stage at diagnosis for the patients. We classified the studies as 'advanced stage' or 'mixed stages.' Advanced stage refers to study populations in which all patients had metastatic CRC, and distant metastases. Mixed stages refers to studies with a combination of patients from Stage I to Stage IV at diagnosis.

Testing was classified by 'completeness.' MSI testing was considered complete if at least the 5 marker NCI panel was tested according to NCI standards. Testing using fewer markers was considered incomplete. For IHC, testing was considered complete if the presence of proteins for all four genes (MLH1, MSH2, MSH6, and PMS2) was evaluated, and incomplete if one to three proteins were tested.

Regimens that included 5FU alone or with leucovorin only were classified as single agent treatment. Regimens that included 5FU in combination with other therapies, such as (but not limited to) FOLFOX, FOLFIRI, or CAPOX were classified as multi-drug treatment.

Studies were classified depending on the type of cancer for the patient, including colon only, rectal only, and colon/rectal combined.

When possible, we recorded outcomes data from multivariate analyses that adjusted for other potentially

\section{Quality categories}

- Good: Studies with a low risk of bias and minimal concerns of applicability

- Fair+: Studies with some risk of bias or concerns regarding applicability; testing does meet NIH standards

- Fair -: Studies with some risk of bias or concerns regarding applicability; testing does not meet NIH standards

- Poor: Studies with a significant risk of bias or greater concerns regarding applicability confounding variables. If only univariate analysis was reported, we recorded these results instead, but also retained an indicator of which analysis is reported.

\section{Statistical analysis}

The hazard ratio (HR) and 95\% confidence interval was abstracted from each article where possible. In cases where the HR was not reported, the value was estimated using the methods described by Tierney et al. [13] HR analysis involved transforming data to ensure that for all studies a HR lower than 1 referred to improved survival in the group receiving $5 \mathrm{FU}$ chemotherapy compared to the group receiving no treatment. In general, data associated with the longest follow-up was retained; however, in instances where patient groups within the same study had variable lengths of follow up, estimates were made for all groups at the shortest time point to allow all groups equivalent time to accrue events.

We conducted random-effects meta-analyses to estimate the pooled effect size of disease-free survival (DFS) and overall survival (OS). We used the metan procedure in Stata $11.2^{\circ}$ [14] for all meta-analyses, which implements the DerSimonion \& Laird method [15]. Analyses were stratified by patient MSI status (MSI-H versus MSS). To examine the statistical significance of the pooled estimates in different strata, we calculated the difference in the log of the pooled effects and their standard error, and from those statistics calculated a z-score and related p-values. Because the DerSimonian \& Laird methods can underestimate statistical heterogeneity when heterogeneity is high or few studies are being pooled, we ran sensitivity analyses using the Profile Likelihood estimation method [16]. Results were almost identical between the two methods, so DerSimonian \& Laird results are presented in the text and figures. 
We examined the $\mathrm{I}^{2}$ statistic as a measure of statistical heterogeneity. We applied the Cochrane Collaboration's rules of thumb for interpreting $\mathrm{I}^{2}$ : less than 40 percent likely represents unimportant heterogeneity, 30 to 60 percent represents moderate heterogeneity, 50 to 90 percent represents substantial heterogeneity; above 75 percent indicates considerable heterogeneity among the studies [17]. We did not conduct any analyses to assess the presence of publication bias or selective outcome reporting within our included studies.

DFS is defined in various ways throughout the CRC literature. Measures of DFS vary in their beginning time point (diagnosis, randomization, and enrollment) and included endpoints (recurrence, progression, and death). In addition to studies of DFS, other studies reported related surrogate endpoints including recurrence-free survival or time to recurrence. Similar variation exists in the measurement of overall survival (OS) in oncology literature. Some studies of survival include only cancer-specific deaths while others include all-cause deaths. It is unclear whether this lack of consistency between trials may alter the findings of a systematic review [18]. In order to examine the impact of these heterogeneous endpoints, multiple sensitivity analyses were conducted from least to most restrictive definition for DFS and OS. The most restrictive were based on definitions published by the FDA in 2007 for guiding clinical trial endpoints [19]. We also performed a subset analysis to assess the study findings in stage II only.

\section{Results}

\section{Eligible studies}

The literature search identified 815 articles for evaluation against the eligibility criteria. We excluded 624 articles based on review of the abstract, and retrieved and evaluated 191 full text articles. After review, 16 studies were included for qualitative analysis and 14 studies were eligible for meta-analysis (see Excluded Studies list in Additional file 1: Search Strategy). The Literature Flow Diagram is presented in Figure 2.

We excluded 46 studies that only reported on subjects treated with 5FU (with no comparison to an untreated population) that would have otherwise been included. For DFS, there were 6 articles that were retained for the meta-analysis of HR [20-25]. For OS there were 14 articles that were retained for the meta-analysis of $\mathrm{HR}$ [20-33]. Two studies [34,35] are included in qualitative discussion but they did not provide the necessary data to be included in the meta-analysis.

All papers were rated as fair quality with the exception of one paper [33] that was rated as good quality. Two papers were excluded from this review based on poor quality [36,37]. Lukish et al. [36] was considered poor quality because the MSI testing was not performed according to the NCI standards [3], all patients were less than 40 years old so the population was not representative of a general population, and the study had a small sample size. Bertagnolli et al. [37] was considered poor quality because the treated and untreated groups were decided by stage and therefore the difference in outcomes is likely highly confounded in terms of prognosis. The fair quality papers were divided into two groups based on whether the MSI testing met NCI standards $[20,21,23,25,28-30,34,35]$ or not $[22,24,26,27,31,32]$.

\section{Microsatellite instability (MSI)}

Overall, nine studies were classified as having complete testing for MSI status [20,21,23,25,28,29,33-35]. Ten studies used MSI testing only [20,23,25-29,33-35], 4 studies used IHC testing only [24,30-32], and 2 studies used both MSI and IHC testing to determine MSI status $[21,22]$. For the purposes of this analysis, subjects were classified as either MSI high (MSI-H) or MSI low or stable (MSS). The mean percentage of MSI-H subjects was $15 \%$ for the included studies, which is comparable to the expected percentage of MSI-H based on population estimates [38].

\section{Study characteristics}

Characteristics of the eligible studies are provided in Table 2. There were a total of 9,312 subjects included across all studies. All of the studies included for the final analysis used treatment regimens that included $5 \mathrm{FU}$ as a single agent. One study also included a separate analysis of a small subset of patients $(n=16)$ that received 5FU, leucovorin, and oxaliplatin (FOLFOX); however, this sample size is too small to draw a meaningful conclusion [32].

Studies were included in the analysis regardless of patient stage at diagnosis. Although in most cases the proportion of patients at each stage was reported, results were not stratified by stage, so results are reported for all stages combined. An analysis focused on stage II cases is presented after the results that included all stages combined. Most studies included both rectal and colon cancer.

\section{MSI status and effect of 5FU treatment based on disease free survival (DFS)}

Forest plots of the effect of 5FU treatment on DFS by MSI status are shown using HRs (Figure 3). For the MSS group, there was an effect of $5 \mathrm{FU}$ treatment on DFS with summary HR of 0.62 (95\% CI: 0.54, 0.71), indicating that patients who received $5 F U$ treatment had longer DFS than patients who did not receive this treatment. We did not detect evidence of heterogeneity across studies for the MSS group for the HR analysis $\left(\mathrm{I}^{2}=7.5 \%, \mathrm{p}=0.37\right)$.

In contrast, the effect of 5FU treatment on DFS was not statistically significant for MSI-H patients with a pooled HR of 0.84 (95\% CI: $0.53,1.32$ ). We did not detect 
evidence of heterogeneity across studies for the MSI-H group $\left(\mathrm{I}^{2}=13.9 \%, \mathrm{p}=0.33\right)$. The sample size for the MSI$\mathrm{H}$ group is smaller than the sample size for the MSS group (651 vs. 4434), and the confidence interval for each study is wider for the MSI-H group compared with the MSS group.

Comparing the summarized point estimates of the effect of 5FU treatment for the MSS group to the MSI-H group, there is not a statistically significant difference $(\mathrm{p}=0.11)$. Thus, we did not find evidence that MSI status affects the likelihood of DFS with 5FU treatment, however data were limited.

One study [35] reported on DFS but could not be included in the quantitative synthesis because it was not possible to estimate the HR using the data available in the publication. In this study, the median DFS was very similar for treated and untreated patients with MSI-H status (34 months versus 36 months, respectively), and for patients with MSS status (24 months versus 25 months, respectively).

We conducted sensitivity analyses to determine whether the results of the analysis are affected by the definition of the DFS outcome. The primary analysis we presented above is the broadest and most inclusive, and incorporated all studies that presented a HR of anything described by the authors as DFS or regression free survival (RFS), which could include substantially different definitions. In sensitivity analyses, we restricted the analysis only to studies

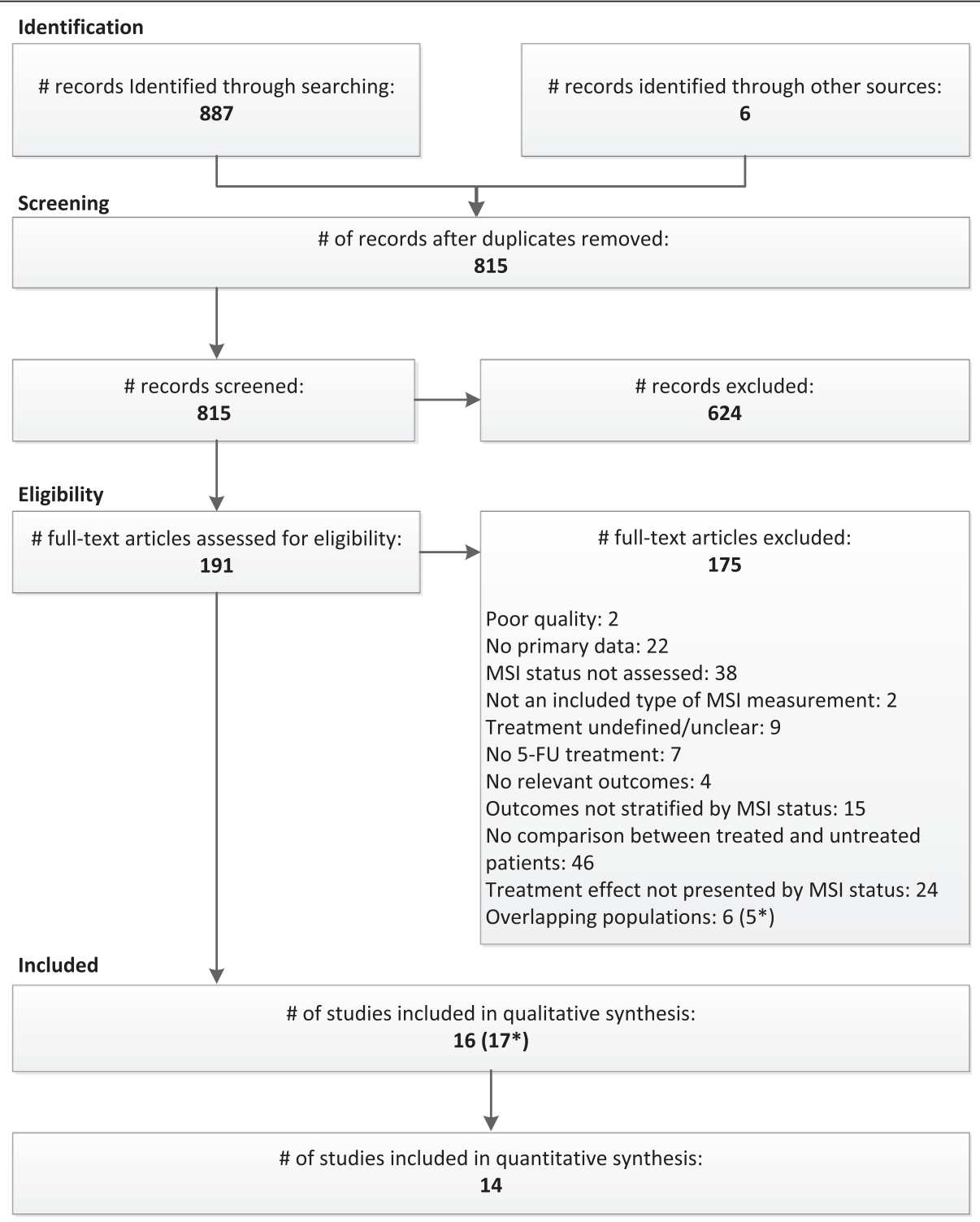

*One study was included in the analysis for stage II only, but was excluded in the full analysis because of overlapping populations

Figure $\mathbf{2}$ Literature flow diagram. 
Table 2 Study characteristics

\begin{tabular}{|c|c|c|c|c|c|c|c|c|c|c|c|}
\hline \multirow[b]{2}{*}{ Study } & \multirow[b]{2}{*}{$\mathbf{N}$} & \multicolumn{4}{|c|}{ Study sample characteristics } & \multirow{2}{*}{$\begin{array}{l}5 \mathrm{FU} \\
\text { Treatment } \\
\text { regimen }\end{array}$} & \multicolumn{3}{|l|}{ MSI assessment } & \multicolumn{2}{|c|}{ Included in HR meta-analysis } \\
\hline & & Tumor & Stage & \% stage II & $\%$ stage IV & & MSI test version & MSI test completeness & MSI-H (\%) & DFS & os \\
\hline Hong 2012 [12] & 947 & Colorectal & Mixed & $40 \%$ & $16.9 \%$ & NR & MSI & Complete & $9 \%$ & $x^{2}$ & $x^{2}$ \\
\hline Hutchins 2011 [11] & 1913 & Colorectal & Mixed & $89 \%$ & $0 \%$ & Single & $\mathrm{IHC}$ & Partial & $11 \%$ & $x^{1}$ & $x^{1}$ \\
\hline Jover 2009 [21] & 496 & Colorectal & Mixed & $59 \%^{3}$ & $0 \%$ & Single & $\mathrm{MSI}+\mathrm{HHC}$ & Complete & $12 \%$ & $x^{2}$ & $x^{2}$ \\
\hline Kim 2007 [25] & 542 & Colon & Mixed & NR & $0 \%$ & Single & MSI & Complete & $18 \%$ & $x^{1}$ & $x^{1}$ \\
\hline Sargent 2010 [22] & 1027 & Colon & Mixed & $52 \%$ & $0 \%$ & Single & $\mathrm{MSI}+\mathrm{IHC}$ & Partial & $16 \%$ & $x^{2}$ & $x^{2}$ \\
\hline Storojeva 2005 [23] & 160 & Colorectal & Mixed & $N R$ & NR & Single & MSI & Complete & $13 \%$ & $x^{2}$ & $x^{2}$ \\
\hline Barratt 2002 [26] & 368 & Colon & Mixed & $61 \%^{3}$ & NR & Single & MSI & Partial & $24 \%$ & & $x$ \\
\hline Benatti 2005 [28] & 1263 & Colorectal & Mixed & $58 \%^{3}$ & $14.6 \%$ & Single & MSI & Complete & $20 \%$ & & $x$ \\
\hline Carethers 2004 [29] & 204 & Colorectal & Mixed & $52 \%$ & $0 \%$ & Single & MSI & Complete & $18 \%$ & & $x^{1}$ \\
\hline Elsaleh 2001 [27] & 732 & Colorectal & Mixed & $0 \%$ & $0 \%$ & Single & MSI & Partial & $9 \%$ & & $x$ \\
\hline Lanza 2006 [30] & 325 & Colorectal & Mixed & $0 \%$ & $0 \%$ & Single & $\mathrm{HC}$ & Partial & $13 \%$ & & $x^{1}$ \\
\hline Liang 2002 [33] & 244 & Colorectal & Advanced & $0 \%$ & $100 \%$ & Single & MSI & Complete & $21 \%$ & & $x^{1}$ \\
\hline Ohrling 2010 [31] & 718 & Colorectal & Mixed & $50 \%$ & $0 \%$ & Single & $\mathrm{HC}$ & Partial & $20 \%$ & & $x^{1}$ \\
\hline Wangefjord 2013 [32] & 112 & Colorectal & Mixed & $0 \%$ & $0 \%$ & Single & $\mathrm{HC}$ & Partial & $15 \%$ & & $x^{1}$ \\
\hline Colombino 2002 [35] & 91 & Rectal & Mixed & $42 \%$ & $N R$ & Single & MSI & Complete & $19 \%$ & & \\
\hline Dietmaier 2006 [34] & 170 & Colon & Mixed & $0 \%$ & $0 \%$ & Single & MSI & Complete & $14 \%$ & & \\
\hline Ribic & 570 & Colon & Mixed & $55 \%$ & $0 \%$ & Single & MSI & Partial & $17 \%$ & & \\
\hline Total & 9312 & & & & & & & & $15 \%$ & & \\
\hline
\end{tabular}

( $5 \mathrm{FU}=5$ fluorouracil; DFS = disease free survival; $\mathrm{OS}=$ overall survival; $\mathrm{HR}=$ hazard ratio; $\mathrm{NR}=$ not reported; $\mathrm{MSI}=$ microsatellite instability; $\mathrm{IHC}=$ immunohistochemistry; $\mathrm{X}=$ study was included in the analysis). 'Hazard ratio had to be estimated from study (see Methods).

${ }^{2}$ Multivariate hazard ratio used in analysis.

${ }^{3}$ Percentage stage II estimated from data reported on full population prior to exclusions for the final analysis data set. 
that include death as an event for DFS and used the FDA definition for DFS [21,22]. When only these two studies are included, there is a statistically significant difference in the effect of 5FU treatment on DFS based on MSI status $(\mathrm{p}=0.008)$. The pooled HRs were quite different, however data was limited to only two studies with only 225 persons with MSI-H (MSI-H:pooled HR $=1.38$ (95\% CI: $0.77-$ 2.49), MSS: $\mathrm{HR}=0.60$ (95\% CI: $0.42-0.85$ ), data not shown).

\section{MSI status and effect of 5FU treatment based on overall survival (OS)}

Forest plots of the effectiveness of 5FU treatment on OS by MSI status are shown using the HR (Figure 4). 5FU treatment was effective in increasing OS in the MSS group with a summary HR of 0.65 (95\% CI: 0.54, 0.79), indicating that patients who received 5FU treatment had longer OS than patients who did not receive this treatment. There was evidence of substantial heterogeneity across studies for the MSS group $\left(\mathrm{I}^{2}=79 \%, \mathrm{p}<0.001\right)$. The largest estimate of the effect of treatment was seen in the study with the shortest followup [20]. In general, there appeared to be a trend of decreasing benefit as the years of observation increased.

The effect of 5FU treatment on OS was very similar for MSI-H patients with a pooled HR of 0.66 (95\% CI: 0.43, 1.03), although the difference between those treated and not treated with $5 \mathrm{FU}$ was not statistically significant in this group. There was evidence of substantial heterogeneity across studies for the MSI-H group $\left(\mathrm{I}^{2}=66 \%, \mathrm{p}<0.001\right)$. This heterogeneity across studies may be driven by lower estimates of the effect of treatment for two studies [27,33], and in general there were relatively small sample sizes with fewer than 100 observations per study in most cases. The confidence intervals around the point estimates for each study are wider for the MSI-H group compared with the MSS group, reflecting the smaller number of patients with MSI-H status (e.g., overall $\mathrm{N}=1293$ vs. 6685 for MSI-H and MSS, respectively).

Not surprisingly, the pooled estimates the effect of 5FU treatment were not statistically different between the two groups $(\mathrm{p}=0.45)$. Thus, it is unlikely the $5 F U$ treatment has a differential effect on OS based on MSI status, however uncertainty remains due to the relatively few events in the MSI-H group and the wide variability in HRs in that group. We note that effect sizes were smaller for MSI-H than MSS patients in all but two of the studies with follow-up of 8 years or less, though differences were not statistically significant. Given the small number of events, these results are consistent with either a reduced likelihood of improved OS for MSI-H patients or no difference between groups.

Two studies [34,35] reported on OS that could not be included in the quantitative synthesis because it was not

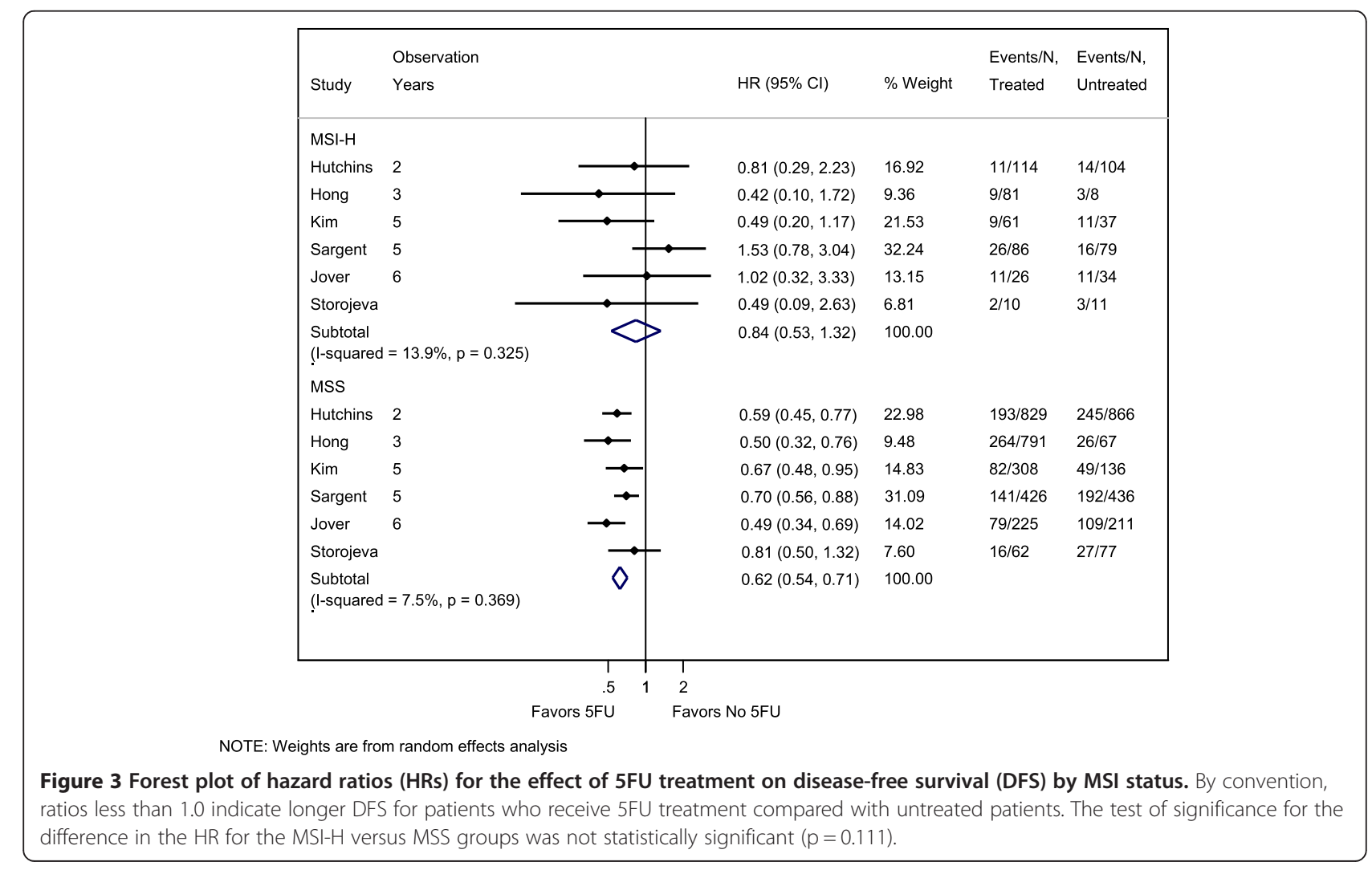




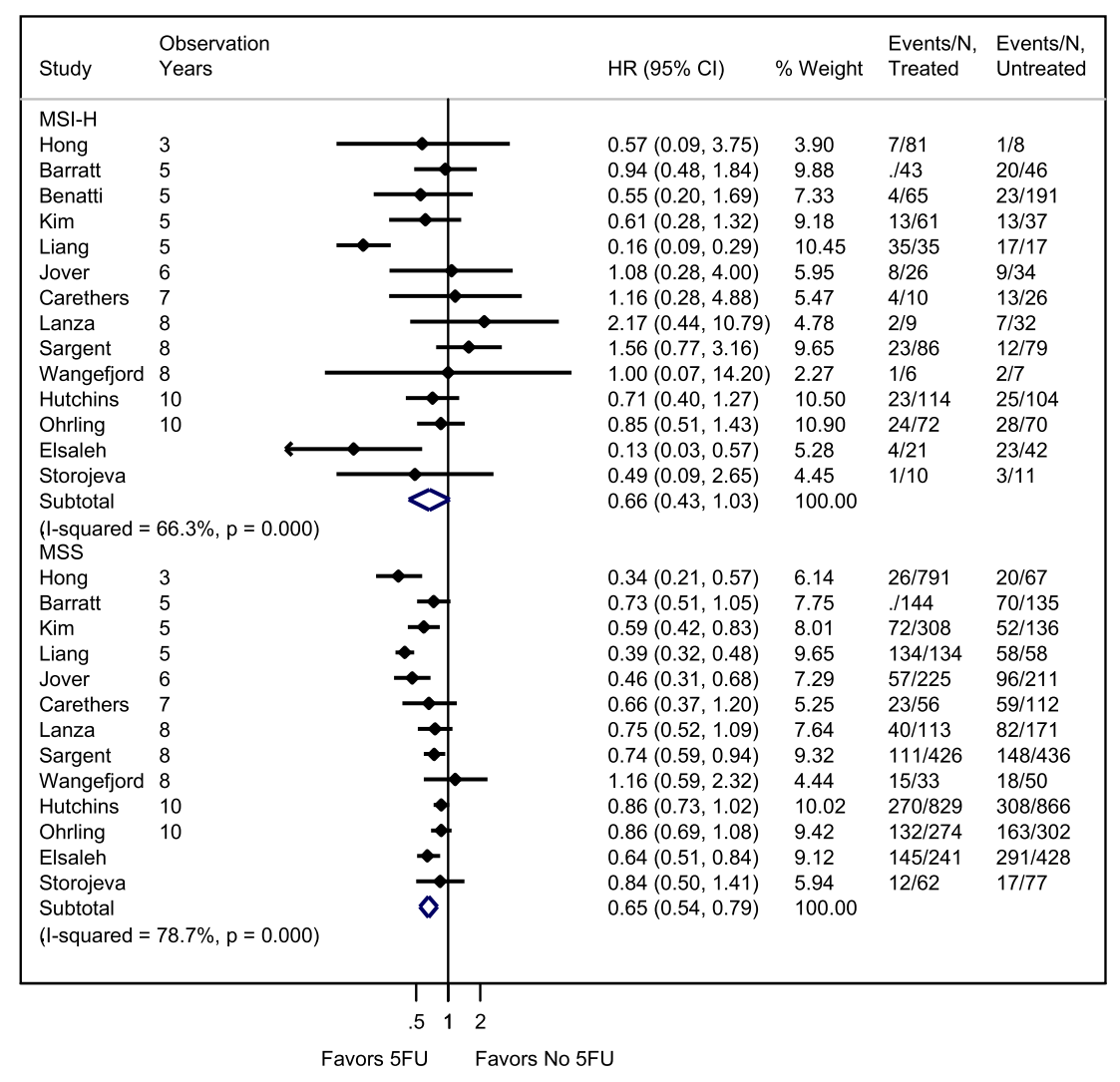

NOTE: Weights are from random effects analysis

Figure 4 Forest plot of hazard ratios (HRs) for the effect of 5FU treatment on overall survival (OS) by MSI status. By convention, ratios less than 1.0 indicate longer OS for patients who receive 5FU treatment compared with untreated patients. The test of significance for the difference in HR for MSI-H versus MSS groups was not statistically significant $(p=0.45)$. Benatti et al. [28] was included in the figure and point estimate, but this study was removed for calculation of p-values, since it contributed data only to the MSI-H group.

possible to estimate the HR using the data available in the publications. In one study, the median OS was very similar for treated and untreated patients with MSI-H status (both 37 months), and for patients with MSS status (26 months versus 28 months, respectively). In the second study, patients who received treatment with $5 \mathrm{FU}$ had fewer events compared with untreated patients for MSI-H (OR = 0.40; 95\% CI: $0.07-2.34)$ and MSS (OR = 0.45 ; $95 \%$ CI: 0.23 - 0.90) patients, with a median follow-up of 44.5 months.

As with DFS, we conducted sensitivity analyses to determine whether the results of the analysis were affected by definition of the OS outcome. In sensitivity analyses, we used increasingly restrictive definitions: 1) only studies that use all-cause mortality [20-23,25,26,31,33] 2) only studies that use cancer-specific survival [27,28,32] and 3) only studies that use the FDA definition of OS $[21,22,24,26,33]$. These analyses did not change the findings, except that in the analysis of studies that use cancerspecific survival the pooled HR estimate for the MSS group was no longer statistically significantly different from 1.0, presumably because of the small sample size with only two studies contributing to the analysis [data not shown].

\section{MSI status and effect of 5FU treatment in stage II cases only}

We assessed the relationship between MSI status and treatment with 5FU in stage II cases only, because the $\mathrm{NCCN}$ guidelines recommend MSI testing for treatment decisions only in this group [6]. There was very limited data available to address this question, since most published studies conducted a joint analysis of mixed stages, and did not provide stratified data by stage. We found three studies that provide data on stage II cases only with a total of 1319 subjects [22,28,39], and one study where the study population included a very high percentage (91\%) of stage II cases with a total of 1708 subjects (Table 2) [24]. Because the data did not use consistent measures across studies, we did not perform a metaanalysis. Instead, we provide a qualitative description of the results.

Ribic et al. [39] reported no difference in OS in MSS patients $(\mathrm{HR}=0.67 ; 95 \% \mathrm{CI} 0.39-1.15)$, or in MSI-H 
patients $(\mathrm{HR}=3.28$; 95\% CI $0.86-12.48)$ with 7.4 years of follow-up. Sargent et al. [22] reported similar findings for DFS, with no difference in DFS for MSS patients $(\mathrm{HR}=0.84 ; 95 \%$ CI $0.57-1.24 ; \mathrm{p}=0.38)$ or for MSI-H patients $(\mathrm{HR}=2.30 ; 95 \%$ CI $0.84-6.24 ; \mathrm{p}=0.09)$ depending on treatment status. For the OS outcome, they reported that the conclusions were similar (data not shown) except that for MSI-H patients the 5FU treated group had decreased OS compared with surgery alone $(\mathrm{HR}=2.95 ; 95 \%$ CI $1.02-8.54 ; \mathrm{p}=0.04)$. Benatti et al. [28] reported no difference in OS for MSS patients (log rank $=2 ; \mathrm{p}=0.151)$ or for MSI-H patients $(\log$ rank = $0.97 ; \mathrm{p}=0.323$ ) depending on $5 \mathrm{FU}$ treatment status with 5 years of follow-up. Hutchins et al. [24] reported a difference in DFS for MSS patients (HR 0.59; 95\% CI $0.45-$ 0.77 ), but not for MSI-H patients ( $\mathrm{HR}=0.81 ; 95 \%$ CI 0.29 - 2.33) with 10 years of follow-up [Figure 2]. For OS, there was not difference in either group based on MSI status [Figure 3]. Consistent with the findings for all stages combined, these studies suggest a potential trend towards larger beneficial effects of treatment in MSS patients compared with MSI-H patients, although the differences are not statistically significant.

In order to incorporate data from as many studies as possible, we also considered all of the studies ranked by percentage of stage II patients. We did not observe a pattern suggesting a relationship between the percentage of stage II patients and response to 5FU by MSI status (see Additional file 2: Figure S1 \& Additional file 3: Figures S2).

\section{Discussion}

We assessed MSI status as a predictive marker for health outcomes in patients undergoing treatment with 5FUbased chemotherapy. The results of this meta-analysis showed that treatment with 5FU-based chemotherapy improved DFS and overall survival in MSS patients and there was a statistically non-significant trend towards better survival in MSI-H patients with 5FU treatment, and no clear difference in the effect of treatment based on MSI status.

Our results expand upon the results of a previous meta-analysis of the same topic by Guastadisegni et al. [40], which included a total sample size of 3121, compared with 9312 subjects in the present analysis. All of the studies reported in the previous analysis are included in our analysis, with the exception of one study [39] that had an overlapping population with a larger and more recent study that was included in our analysis [22]. The previous analysis also differed from ours in the statistical procedures used to compute the summary statistic. We used methods to estimate the HR [13], which takes into account the number and timing of events, and censoring. Odds ratios (ORs) take into account only the number of events, and can produce biased estimates for time-to-event outcomes. The authors of the previous analysis on this topic concluded that whereas they could establish MSI status as a favorable prognostic marker based on DFS (summary OR $=0.58,95 \%$ CI $0.47-0.72$ ) and OS (summary OR $=0.6$; 95\% CI $0.53-0.69$ ) for CRC patients, they had inconclusive results for MSI status as a predictive marker of response to treatment with 5FU chemotherapy. Similar to our analysis, they determined that there was a significant beneficial effect of 5FU chemotherapy in patients with MSS tumors (OR = 0.52 ; $95 \%$ CI $0.4-0.6)$. However, the sample size for patients with MSI-H tumors in their analysis was small $(n=396)$. The point estimate for the effect of 5FU treatment in MSI-H patients was similar to what we found $(\mathrm{OR}=0.69$; 95\% CI $0.3-1.5)$ for OS, and there was significant heterogeneity across studies $(\mathrm{p}=0.03)$, so no clear conclusion could be reached. Our analysis included a larger population of 1293 MSI-H patients; however, statistically significant heterogeneity was still identified in the overall survival analysis.

Des Guetz et al. [41] also reported a meta-analysis on this topic that included seven studies with 3690 patients combined, six of which were reported by Guastadisegni et al. [40] All patients included in these studies are Stage II or Stage III CRC cases. Given this overlap, not surprisingly they found a beneficial effect of chemotherapy among MSS patients, but no benefit of 5FU chemotherapy among MSI-H patients for Regression-Free Survival $(H R=0.96$; $95 \%$ CI $0.62-1.49)$ or OS $(H R=$ $0.70,95 \%$ CI $0.44-1.09)$. In contrast to the conclusions of Guastadisegni et al. [40], these authors concluded that MSI-H status is a predictive factor of non-response to 5FU chemotherapy based on a meta-analysis for the interaction between MSI status and treatment effect, suggesting a lesser benefit for MSI-H patients compared with MSS patients $((\mathrm{HR}=0.77,95 \%$ CI $0.68-0.87)$.

A previous study addressed this question and reached seemingly different conclusions. Des Guetz et al. [42] conducted a meta-analysis restricted only to patients with metastatic CRC who received treatment with 5FU chemotherapy. The results were reported in terms of the response rate ratio using the RECIST criteria [43]. The authors found that among patients treated with $5 \mathrm{FU}$ chemotherapy, the response rate ratio was 0.82 (95\% CI $0.65-1.03$ ), where ratios less than 1.0 indicate that the response rate to chemotherapy was better among MSI-H patients compared with MSS patients. However, the authors concluded that MSI status does not predict the effect of 5FU chemotherapy. When we repeated this analysis with existing studies [33,44-54], however, we had a statistically significant finding. For studies in which patients were treated with $5 \mathrm{FU}$ as a single agent, the summary RR is 0.52 (95\% CI $0.40-0.68)$, indicating that 
MSI-H patients have a better response to $5 \mathrm{FU}$ chemotherapy than MSS patients (see Additional file 4: Figure S3). This apparent discrepancy can be understood by considering Figure 1, which shows the expected impact of MSI status as both a prognostic and predictive factor. By evaluating only patients who received 5FU treatment, we cannot untangle the effects of prognosis and treatment. Interestingly, in this analysis we observed a substantially different effect for studies in which patients were treated with $5 \mathrm{FU}$ as a single agent versus studies in which patients received 5FU as part of a combination therapy. Unfortunately, none of the studies in our primary meta-analysis used combination therapy, so we were unable to confirm whether this effect remained using different outcomes (DFS, OS) or subjects with different stages of CRC.

The data that we report do not support the recommendation for determination of MSI status in stage II CRC patients. One guideline recommends determination of MSI status for all patients with stage II disease because stage II MSI-H patients may have good prognosis and do not benefit from 5FU treatment [6]. This guideline only cites the results of one study [22], which was also reported by us, showing a statistically significant decrease in OS in the treatment group compared with surgery alone in MSI-H stage II patients $(\mathrm{HR}=$ 2.95; 95\% CI $1.02-8.54 ; \mathrm{p}=0.04)$. This was the only study with a statistically significant finding; the others showed no difference for stage II patients in MSI-H patients. Further, this study had the most extreme HR in favor of no 5FU treatment for MSI-H patients among studies that reported on stage II patients (Additional file 2: Figure S1 and Additional file 3: Figure S2).

There were several limitations to our meta-analysis. First, not all studies reported hazard ratios or counts of events, which were the primary data necessary to include the studies in the meta-analysis. In some cases, we were able estimate the HR value using the methods described by Tierney et al. [13]. We conducted a sensitivity analysis by excluding studies in which the data were estimated, and the overall conclusions remained the same (data not shown). Related to this issue, a second limitation of our approach is that when we estimated data using the Kaplan-Meier curves, we selected the longest possible follow-up available to determine the estimated number of events. This approach could lead to potential bias towards the null hypothesis of no difference, because Figures 3 and 4 show a possible trend with the most significant HRs being the ones with the shortest follow-up time.

None of the studies reported in our primary metaanalysis used contemporary treatment regimens that include 5FU in combination therapies, such as FOLFOX or FOLFIRI. This is a strength of the available data, since it is then possible to explore the ability of MSI status to predict 5FU treatment response in isolation, and without contamination of the effects of other treatments given simultaneously. However, it is not clear that these findings represent the effect of MSI status on response to $5 \mathrm{FU}$ in combination therapy.

We did not consider whether molecular subtypes of MSI-H patients experience differential response to $5 \mathrm{FU}$ chemotherapy. Greater heterogeneity of MSI-H tumors is now recognized [55] with various epigenetic and genetic alterations that may alter the prognostic and predictive implications for patients. For instance, recently, large deletions in HSP110 $\mathrm{T}_{17}$ have been associated with improved survival in patients treated with 5FU-based chemotherapy [56,57]. Future analyses may need to account for this heterogeneity.

Finally, despite the increase in sample size for this analysis compared with previously reported metaanalyses on this topic, the number of MSI-H patients available for analysis remains limited, with substantially larger confidence intervals and lower statistical power compared with the findings for the MSS group. This limitation is coupled with an evidence base that relies primarily on retrospective study designs that have a greater potential for selection bias, and substantial (although not significant) heterogeneity of effect sizes reported in studies. One ongoing prospective study was identified in our search for additional clinical trials and meeting abstracts: The Eastern Cooperative Oncology Group (ECOG) E5202 Trial (ClinicalTrials.gov Identifier: NCT00217737) will assign treatment arms for stage II colorectal cancer patients based on MSI status in conjunction with $18 \mathrm{q} \mathrm{LOH}$. Those patients who are considered high risk (based on MSS and 18q LOH) will be randomized to FOLFOX6 with or without bevacizumab. Patients considered low risk due to retention of $18 \mathrm{q}$ alleles or MSI-H status will receive observation alone. While no publications were identified for this study, the clinical trial record indicates that primary data collection is completed.

\section{Conclusions}

We conclude that the results of this meta-analysis do not support the use of MSI status for the direct benefit of the patient in guiding decisions about the use of 5FU treatment for CRC. Testing for MSI status among CRC patients is becoming increasingly common [58], given efforts to implement recommendations for universal screening of newly diagnosed CRC patients to identify cases of Lynch Syndrome [59]. Whereas substantial benefit of MSI testing has been demonstrated in terms of impact on relatives [60] and impact on prognosis [7], there remains limited use of this testing in guiding treatment decisions for patients already diagnosed with CRC. 


\section{Additional files}

\section{Additional file 1: Search strategy used to identify relevant studies in Medline.}

Additional file 2: Figure S1. Forest plot of hazard ratios (HRs) for the effect of 5FU treatment on disease-free survival (DFS) by MSI status ranked by percentage of stage II patients. By convention, ratios less than 1.0 indicate longer DFS for patients who receive 5FU treatment compared with untreated patients. The test of significance for the difference in the HR for the MSI-H versus MSS groups was not statistically significant $(p=0.111)$.

Additional file 3: Figure S2. Forest plot of hazard ratios (HRs) for the effect of 5FU treatment on overall survival (OS) by MSI status ranked by percentage of stage II patients. By convention, ratios less than 1.0 indicate longer OS for patients who receive 5FU treatment compared with untreated patients. The test of significance for the difference in HR for MSI-H versus MSS groups was not statistically significant $(p=0.45)$. Benatti et al. [28] was included in the figure and point estimate, but this study was removed for calculation of p-values, since it contributed data only to the MSI-H group.

Additional file 4: Figure S3. Forest plot of response rate ratio (RR) for the effect of MSI status on response to treatment among patients treated with 5FU therapy. By convention, ratios less than 1.0 indicate a better response to treatment among patients with MSI-L/S status.

\section{Competing interests}

The authors declare that they have no competing interests.

\section{Authors' contributions}

KG, TK, EW all contributed to the development of the research questions, search strategies, abstract and article review, and data abstraction. KG and EO contributed to the statistical analysis. All authors contributed to the synthesis of results and writing of the manuscript. All authors read and approved the final manuscript.

\section{Acknowledgements}

This work was funded by Grant R01 CA140377 from the National Cancer Institute. The study sponsors had no role in the study design, in the collection, analysis and interpretation of data; in the writing of the manuscript; or in the decision to submit the manuscript for publication.

\section{Received: 19 March 2014 Accepted: 20 February 2015}

Published online: 21 March 2015

\section{References}

1. American Cancer Society. What are the key statistics about colorectal cancer? Available at: http://www.cancer.org/cancer/colonandrectumcancer/ detailedguide/colorectal-cancer-key-statistics.

2. Ionov Y, Peinado MA, Malkhosyan S, Shibata D, Perucho M. Ubiquitous somatic mutations in simple repeated sequences reveal a new mechanism for colonic carcinogenesis. Nature. 1993;363(6429):558-61.

3. Boland CR, Thibodeau SN, Hamilton SR, Sidransky D, Eshleman JR, Burt RW, et al. A National Cancer Institute Workshop on Microsatellite Instability for cancer detection and familial predisposition: development of international criteria for the determination of microsatellite instability in colorectal cancer. Cancer Res. 1998;58(22):5248-57.

4. Lindor NM, Burgart $\mathrm{L}$, Leontovich $\mathrm{O}$, Goldberg RM, Cunningham JM, Sargent DJ, et al. Immunohistochemistry versus microsatellite instability testing in phenotyping colorectal tumors. J Clin Oncol. 2002;20(4):1043-8.

5. Chu HY, Zheng QC, Zhao YS, Zhang HX. Homology modeling and molecular dynamics study on N-acetylneuraminate lyase. J Mol Model. 2009;15(3):323-8.

6. National Comprehensive Cancer Network. NCCN clinical practice guidelines in oncology: colon cancer (version 1.2013). Available at: www.ncen.org.

7. Popat S, Hubner R, Houlston RS. Systematic review of microsatellite instability and colorectal cancer prognosis. [Review] [86 refs]. J Clin Oncol. 2005;23(3):609-18.

8. Sturgeon CM, Duffy MJ, Stenman UH, Lilja H, Brunner N, Chan DW, et al. National Academy of Clinical Biochemistry laboratory medicine practice guidelines for use of tumor markers in testicular, prostate, colorectal, breast, and ovarian cancers. Clin Chem. 2008;54(12):e11-79.
9. Duffy MJ, van Dalen A, Haglund C, Hansson L, Holinski-Feder E, Klapdor R, et al. Tumour markers in colorectal cancer: European group on tumour markers (EGTM) guidelines for clinical use. Eur J Cancer. 2007:43(9):1348-60

10. Locker GY, Hamilton S, Harris J, Jessup JM, Kemeny N, Macdonald JS, et al. ASCO 2006 update of recommendations for the use of tumor markers in gastrointestinal cancer. J Clin Oncol. 2006;24(33):5313-27.

11. Whiting PF, Rutjes AW, Westwood ME, Mallett S, Deeks JJ, Reitsma JB, et al. QUADAS-2: a revised tool for the quality assessment of diagnostic accuracy studies. Ann Intern Med. 2011;155(8):529-36.

12. Juni $P$, Witschi $A$, Bloch $R$, Egger $M$. The hazards of scoring the quality of clinical trials for meta-analysis. JAMA. 1999;282(11):1054-60.

13. Tierney JF, Stewart LA, Ghersi D, Burdett S, Sydes MR. Practical methods for incorporating summary time-to-event data into meta-analysis. Trials. 2007:8:16.

14. Bradburn MJ, Deeks JJ, Altman DG. Metan-a command for meta-analysis in Stata. Meta-analysis in Stata: an updated collection from the Stata Journal. College Station, TX: Stata Press, 1998. p. 3-28.

15. DerSimonian R, Laird N. Meta-analysis in clinical trials. Control Clin Trials. 1986;7(3):177-88.

16. Kontopantelis $\mathrm{E}$, Reeves D. Performance of statistical methods for meta-analysis when true study effects are non-normally distributed: A simulation study. Stat Methods Med Res. 2012;21(4):409-26.

17. The Cochrane Collaboration. Cochrane Handbook for Systematic Reviews of Interventions. Sussex: Wiley; 2011.

18. Punt $\mathrm{CJ}$, Buyse $\mathrm{M}$, Kohne $\mathrm{CH}$, Hohenberger $\mathrm{P}$, Labianca $\mathrm{R}$, Schmoll HJ, et al. Endpoints in adjuvant treatment trials: a systematic review of the literature in colon cancer and proposed definitions for future trials. J Natl Cancer Inst. 2007;99(13):998-1003.

19. U.S.Department of Health and Human Services Food and Drug Administration Center for Drug Evaluation and Research (CDER) Center for Biologics Evaluation and Research (CBER). Guidance for Industry Clinical Trial endpoints for the approval of cancer drugs and biologics. Available at: http://www.fda.gov/downloads/Drugs/GuidanceComplianceRegulatory Information/Guidances/ucm071590.pdf. Accessed on: 7-30-2013.

20. Hong SP, Min BS, Kim TI, Cheon JH, Kim NK, Kim H, et al. The differential impact of microsatellite instability as a marker of prognosis and tumour response between colon cancer and rectal cancer. Eur J Cancer. 2012:48(8):1235-43.

21. Jover R, Zapater P, Castells A, Llor X, Andreu M, Cubiella J, et al. The efficacy of adjuvant chemotherapy with 5-fluorouracil in colorectal cancer depends on the mismatch repair status. Eur J Cancer. 2009;45(3):365-73.

22. Sargent DJ, Marsoni S, Monges G, Thibodeau SN, Labianca R, Hamilton $S R$, et al. Defective mismatch repair as a predictive marker for lack of efficacy of fluorouracil-based adjuvant therapy in colon cancer. [Erratum appears in J Clin Oncol. 2010 Oct 20;28(30):4664]. J Clin Oncol. 2010;28(20):3219-26.

23. Storojeva I, Boulay JL, Heinimann K, Ballabeni P, Terracciano L, Laffer U, et al Prognostic and predictive relevance of microsatellite instability in colorectal cancer. Oncol Rep. 2005;14(1):241-9.

24. Hutchins G, Southward K, Handley K, Magill L, Beaumont C, Stahlschmidt J, et al. Value of mismatch repair, KRAS, and BRAF mutations in predicting recurrence and benefits from chemotherapy in colorectal cancer.[Erratum appears in J Clin Oncol. 2011 Jul 20;29(21):2949]. J Clin Oncol. 2011;29(10):1261-70

25. Kim GP, Colangelo LH, Wieand HS, Paik S, Kirsch IR, Wolmark N, et al. Prognostic and predictive roles of high-degree microsatellite instability in colon cancer, a National Cancer Institute-National Surgical Adjuvant Breast and Bowel Project Collaborative Study. J Clin Oncol. 2007;25(7):767-72

26. Barratt PL, Seymour MT, Stenning SP, Georgiades I, Walker C, Birbeck K, et al. UKCCCR AXIS trial collaborators. Adjuvant. DNA markers predicting benefit from adjuvant fluorouracil in patients with colon cancer, a molecular study. Lancet. 2002;360(9343):1381-91.

27. Elsaleh H, Powell B, McCaul K, Grieu F, Grant R, Joseph D, et al. P53 alteration and microsatellite instability have predictive value for survival benefit from chemotherapy in stage III colorectal carcinoma. Clin Cancer Res. 2001;7(5):1343-9.

28. Benatti P, Gafa R, Barana D, Marino M, Scarselli A, Pedroni M, et al. Microsatellite instability and colorectal cancer prognosis. [Erratum appears in Clin Cancer Res. 2006 Jun 15;12(12):3868-9]. Clin Cancer Res. 2005;11(23):8332-40. 
29. Carethers JM, Smith EJ, Behling CA, Nguyen L, Tajima A, Doctolero RT, et al. Use of 5-fluorouracil and survival in patients with microsatellite-unstable colorectal cancer. Gastroenterology. 2004;126(2):394-401.

30. Lanza G, Gafa R, Santini A, Maestri I, Guerzoni L, Cavazzini L. Immunohistochemical test for MLH1 and MSH2 expression predicts clinical outcome in stage II and III colorectal cancer patients. J Clin Oncol. 2006;24(15):2359-67.

31. Ohrling $K$, Edler $D$, Hallstrom M, Ragnhammar $P$. Mismatch repair protein expression is an independent prognostic factor in sporadic colorectal cancer. Acta Oncol. 2010;49(6):797-804.

32. Wangefjord S, Brandstedt J, Lindquist KE, Nodin B, Jirstrom K, Eberhard J. Associations of beta-catenin alterations and MSI screening status with expression of key cell cycle regulating proteins and survival from colorectal cancer. Diagn Pathol. 2013;8:10.

33. Liang JT, Huang KC, Lai HS, Lee PH, Cheng YM, Hsu HC, et al. High-frequency microsatellite instability predicts better chemosensitivity to high-dose 5-fluorouracil plus leucovorin chemotherapy for stage IV sporadic colorectal cancer after palliative bowel resection. Int J Cancer. 2002;101(6):519-25.

34. Dietmaier W, Bettstetter M, Wild PJ, Woenckhaus M, Rummele P, Hartmann A, et al. Nuclear Maspin expression is associated with response to adjuvant 5-fluorouracil based chemotherapy in patients with stage III colon cancer. Int J Cancer. 2006;118(9):2247-54.

35. Colombino M, Cossu A, Manca A, Dedola MF, Giordano M, Scintu F, et al. Prevalence and prognostic role of microsatellite instability in patients with rectal carcinoma. Ann Oncol. 2002;13(9):1447-53.

36. Lukish JR, Muro K, DeNobile J, Katz R, Williams J, Cruess DF, et al. Prognostic significance of DNA replication errors in young patients with colorectal cancer. Ann Surg. 1998;227(1):51-6.

37. Bertagnolli MM, Redston M, Compton CC, Niedzwiecki D, Mayer RJ, Goldberg RM, et al. Microsatellite instability and loss of heterozygosity at chromosomal location 18q: prospective evaluation of biomarkers for stages II and III colon cancer-a study of CALGB 9581 and 89803. J Clin Oncol. 2011;29(23):3153-62

38. Kinzler KW, Vogelstein B. Lessons from hereditary colorectal cancer. Cell. 1996;87(2):159-70.

39. Ribic CM, Sargent DJ, Moore MJ, Thibodeau SN, French AJ, Goldberg RM, et al. Tumor microsatellite-instability status as a predictor of benefit from fluorouracil-based adjuvant chemotherapy for colon cancer. N Engl J Med. 2003;349(3):247-57.

40. Guastadisegni C, Colafranceschi M, Ottini L, Dogliotti E. Microsatellite instability as a marker of prognosis and response to therapy a meta-analysis of colorectal cancer survival data. [Review]. Eur J Cancer. 2010;46(15):2788-98.

41. Des Guetz G, Schischmanoff O, Nicolas P, Perret GY, Morere JF, Uzzan B. Does microsatellite instability predict the efficacy of adjuvant chemotherapy in colorectal cancer? A systematic review with meta-analysis. [Review] [36 refs]. Eur J Cancer. 2009;45(10):1890-6.

42. Des Guetz G, Uzzan B, Nicolas P, Schischmanoff O, Perret GY, Morere JF. Microsatellite instability does not predict the efficacy of chemotherapy in metastatic colorectal cancer. A systematic review and meta-analysis. [Review] [26 refs]. Anticancer Res. 2009;29(5):1615-20.

43. Therasse P, Arbuck SG, Eisenhauer EA, Wanders J, Kaplan RS, Rubinstein L, et al. New guidelines to evaluate the response to treatment in solid tumors. European Organization for Research and Treatment of Cancer, National Cancer Institute of the United States, National Cancer Institute of Canada. J Natl Cancer Inst. 2000;92(3):205-16.

44. Bendardaf R, Lamlum H, Ristamaki R, Korkeila E, Syrjanen K, Pyrhonen S. Mismatch repair status is a predictive factor of tumour response to 5-fluorouracil and irinotecan chemotherapy in patients with advanced colorectal cancer. Tumour Biol. 2007;28(4):212-20.

45. Brueckl WM, Moesch C, Brabletz T, Koebnick C, Riedel C, Jung A, et al. Relationship between microsatellite instability, response and survival in palliative patients with colorectal cancer undergoing first-line chemotherapy. Anticancer Res. 2003;23(2C):1773-7.

46. Charara M, Edmonston TB, Burkholder S, Walters R, Anne P, Mitchell E, et al. Microsatellite status and cell cycle associated markers in rectal cancer patients undergoing a combined regimen of 5-FU and CPT-11 chemotherapy and radiotherapy. Anticancer Res. 2004;24(5B):3161-7.

47. Chua W, Goldstein D, Lee CK, Dhillon H, Michael M, Mitchell P, et al. Molecular markers of response and toxicity to FOLFOX chemotherapy in metastatic colorectal cancer. Br J Cancer. 2009;101(6):998-1004.
48. Des Guetz G, Mariani P, Cucherousset J, Benamoun M, Lagorce C, Sastre X, et al. Microsatellite instability and sensitivitiy to FOLFOX treatment in metastatic colorectal cancer.[Erratum appears in Anticancer Res. 2007 Sep-Oct;27 (5b):3667]. Anticancer Res. 2007;27(4C):2715-9.

49. Ismail AS, Quinn MG, Wright MA, Ernst A, Kao V, Grogan L, et al. A phase II and pharmacologic study of fluorouracil given by a 1-hour infusion daily for 5 days with leucovorin and interferon alpha-2a in adenocarcinoma of the large bowel. Oncol Rep. 2005;13(6):1145-52.

50. Kim JE, Hong YS, Ryu MH, Lee JL, Chang HM, Lim SB, et al. Association between deficient mismatch repair system and efficacy to irinotecancontaining chemotherapy in metastatic colon cancer. Cancer Sci. 2011;102(9):1706-11.

51. Kim ST, Lee J, Park SH, Park JO, Lim HY, Kang WK, et al. The effect of DNA mismatch repair (MMR) status on oxaliplatin-based first-line chemotherapy as in recurrent or metastatic colon cancer. Med Oncol. 2010;27(4):1277-85.

52. Koopman M, Kortman GA, Mekenkamp L, Ligtenberg MJ, Hoogerbrugge N, Antonini NF, et al. Deficient mismatch repair system in patients with sporadic advanced colorectal cancer. Br J Cancer. 2009;100:266-73.

53. Muller Cl, Schulmann K, Reinacher SA, Andre N, Arnold D, Tannapfel A, et al. Predictive and prognostic value of microsatellite instability in patients with advanced colorectal cancer treated with a fluoropyrimidine and oxaliplatin containing first-line chemotherapy. A report of the AIO Colorectal Study Group. Int J Colorectal Dis. 2008;23:1033-9.

54. Rosty C, Chazal M, Etienne MC, Letoublon C, Bourgeon A, Delpero JR, et al. Determination of microsatellite instability, p53 and K-RAS mutations in hepatic metastases from patients with colorectal cancer: relationship with response to 5-fluorouracil and survival. Int J Cancer. 2001;95(3):162-7.

55. Kim JH, Kang GH. Molecular and prognostic heterogeneity of microsatelliteunstable colorectal cancer. World J Gastroenterol. 2014;20(15):4230-43.

56. Kim JH, Kim KJ, Rhee YY, Oh S, Cho NY, Lee HS, et al. Expression status of wild-type HSP110 correlates with HSP110 T17 deletion size and patient prognosis in microsatellite-unstable colorectal cancer. Mod Pathol. 2014;27(3):443-53.

57. Collura A, Lagrange A, Svrcek M, Marisa L, Buhard O, Guilloux A, et al. Patients with colorectal tumors with microsatellite instability and large deletions in HSP110 T17 have improved response to 5-fluorouracil-based chemotherapy. Gastroenterology. 2014;146(2):401-11.

58. Cohen SA. Current lynch syndrome tumor screening practices: a survey of genetic counselors. J Genet Couns. 2014;23(1):38-47.

59. Recommendations from the EGAPP Working Group. Genetic testing strategies in newly diagnosed individuals with colorectal cancer aimed at reducing morbidity and mortality from Lynch syndrome in relatives. Genet Med. 2009;11(1):35-41.

60. Ladabaum U, Wang G, Terdiman J, Blanco A, Kuppermann M, Boland CR, et al. Strategies to identify the Lynch syndrome among patients with colorectal cancer: a cost-effectiveness analysis. Ann Intern Med. 2011;155(2):69-79.

\section{Submit your next manuscript to BioMed Central and take full advantage of:}

- Convenient online submission

- Thorough peer review

- No space constraints or color figure charges

- Immediate publication on acceptance

- Inclusion in PubMed, CAS, Scopus and Google Scholar

- Research which is freely available for redistribution 